14,19

\title{
Фазовые переходы в молекулярных кристаллах н-алканов: три-, тетра- и пентакозане
}

\author{
() В.М. Егоров, В.А. Марихин, Л.П. Мясникова, П.Н. Якушев \\ Физико-технический институт им. А.Ф. Иофрфе РАН, \\ Санкт-Петербург, Россия \\ E-mail: victor_egorov1@inbox.ru
}

(Поступила в Редакцию 27 марта 2017 г.)

\begin{abstract}
Методом дифференциальной сканирующей калориметрии исследованы фазовые переходы в нормальных алканах: трикозане $\left(\mathrm{C}_{23} \mathrm{H}_{48}\right)$, тетракозане $\left(\mathrm{C}_{24} \mathrm{H}_{50}\right)$ и пентакозане $\left(\mathrm{C}_{25} \mathrm{H}_{52}\right)$. Устранение методических погрешностей позволило получить истинные значения термодинамических параметров фазовых переходов и выявить их природу. Проведен сравнительный анализ скачков теплоемкости на основе теории размытых фазовых переходов первого рода.
\end{abstract}

Работа выполнена при финансовой поддержке РФФИ (код проекта № 16-03-00493).

DOI: 10.21883/FTT.2017.10.44978.095

Изучение гомологических рядов различных длинноцепочечных молекулярных кристаллов (н-алканов и их производных) открывает новые возможности для установления количественных закономерностей между структурой и термодинамическими свойствами полимеров, поскольку эти соединения не содержат химических дефектов и могут быть получены с недостижимой для обычных полимеров чистотой.

Одной из важных проблем в физике твердого тела является выяснение ряда дискуссионных вопросов о природе фазовых переходов (ФП) первого и второго рода. Выяснение этих вопросов важно не только с фундаментальной точки зрения, но и для решения многих практически важных задач, поскольку при получении изделий из полимеров всегда происходит ФП на различных этапах их переработки.

Одним из эффективных методов исследования ФП является метод дифференциальной сканирующей калориметрии (ДСК), широко используемый для изучения молекулярных кристаллов различного типа [1-5], в том числе парафинов [6-8]. В большинстве опубликованных работ, как правило, не проводятся и не анализируются исследования в циклах нагревание-охлаждение, которые, как показано в настоящей работе, важны для выявления природы ФП, а также для получения истинных профилей эндо- и экзопиков на термограммах. Кроме того, в подавляющем числе работ не устраняются методические погрешности, связанные с эффектом термосопротивления при ДСК-измерениях, которые могут приводить к существенным искажениям значений термодинамических характеристик ФП. Последнее обстоятельство является очень важным для анализа экспериментальных данных, который может быть проведен на основе развитых в настоящее время теорий размытых ФП первого рода $[9,10]$ и ФП второго рода [11].

В работе исследовались образцы н-алканов с четным и нечетным числом атомов углерода в цепи, что позво- лило выявить (см. далее) влияние эффекта четности на кинетику развития ФП в н-алканах.

Образцы трикозана $\left(\mathrm{C}_{23} \mathrm{H}_{48}\right)$, тетракозана $\left(\mathrm{C}_{24} \mathrm{H}_{50}\right)$ и пентакозана $\left(\mathrm{C}_{25} \mathrm{H}_{52}\right)$ приобретены в фирме „Sigma“. Тепловые свойства исследовались на калориметре DSC 6100 „Seiko“ при варьировании скоростей нагревания и охлаждения в диапазоне $0.6-5 \mathrm{~K} / \mathrm{min}$. Небольшой вес образцов для испытаний, составлявший $3-5 \mathrm{mg}$, обеспечивал минимальное термическое сопротивление калориметрической ячейки и уменьшал методические погрешности.

На рис. 1 представлены кривые ДСК, полученные при нагревании и охлаждении образцов трикозана (1), тетракозана (2) и пентакозана (3). Как видно из рисунка, при нагревании эндотермический ФП из кристаллического

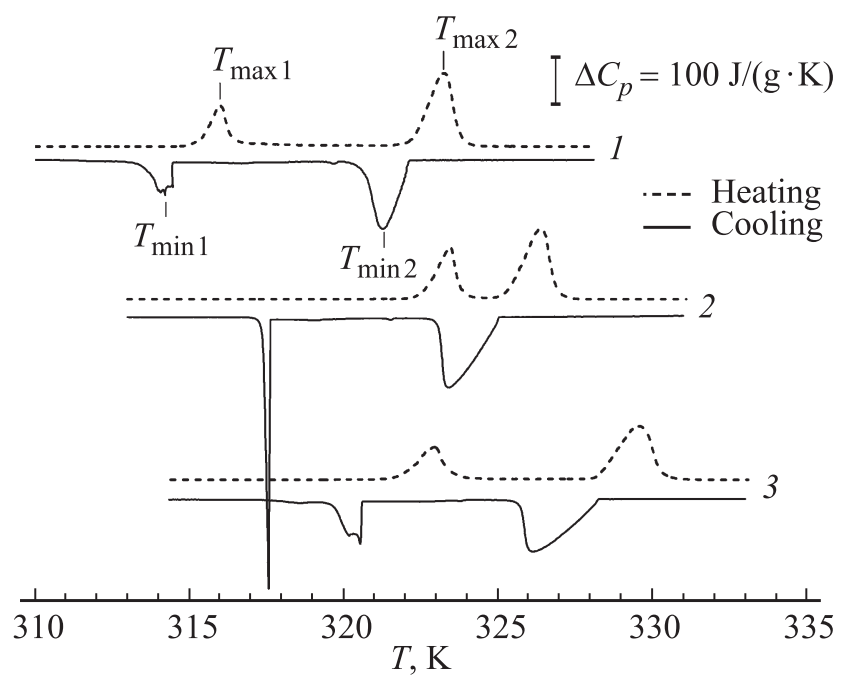

Рис. 1. Кривые ДСК, полученные при нагревании (штриховые линии) и охлаждении (сплошные линии) образцов три- (1) тетра- (2) и пентакозана (3). Скорость сканирования $V=0.6 \mathrm{~K} / \mathrm{min}$. 
состояния в расплав для этих парафинов осуществляется в два этапа: на кривых ДСК виден дублет с температурами максимумов $T_{\max 1}$ и $T_{\max 2}$. Обратный процесс изменения фазового состояния расплав-кристалл при охлаждении происходит также в два этапа (температуры минимумов соответствующих им экзотермических пиков $T_{\min 1}$ и $\left.T_{\min 2}\right)$. Следует отметить, что формы эндотермических пиков перехода из кристаллического состояния в расплав для каждого из исследованных материалов имеют свои особенности, анализ которых приводится далее.

Такая картина в общем виде соответствует результатам опубликованных работ, поэтому можно ожидать, что эндотермический пик с $T_{\max 1}$ при нагревании связан, по-видимому, со структурным фазовым переходом в твердом состоянии $T_{\mathrm{s}-\mathrm{s}}$, за которым следует при температуре $T_{\max 2}$ плавление образца. Соответственно при охлаждении из расплава в этом случае наблюдается обратная картина: при $T_{\min 2}$ происходит их кристаллизация, а при $T_{\min 1}$ - твердофазный переход [6-8].

Для понимания физической природы регистрируемых на термограммах переходов необходимо прояснить картину с наблюдаемым температурным несовпадением пиков. На кривых ДСК видны смещения относительных положений максимумов эндо- и экзопиков в циклах нагревание-охлаждение: $\left(T_{\max 1}-T_{\min 1}\right)$ и $\left(T_{\max 2}-T_{\min 2}\right)$ (так называемый эффект гистерезиса). Важно, что смещения имеют для каждого из пиков дублета свою природу, обусловленную как методическими, так и физическими причинами. Известно [12], что при использовании метода ДСК возникают методические погрешности (смещение пиков), связанные с наличием термосопротивления испытываемого образца в калориметрической ячейке, которое существенно зависит от массы образца и скорости сканирования. В работе [13] показано, что эти методические погрешности могут быть устранены следующим образом: по экспериментальным данным при вариации скоростей сканирования $V$ (нагревания или охлаждения) строятся зависимости $T_{\max , \min }=f\left(V^{1 / 2}\right)$, которые при отсутствии структурных трансформаций должны быть линейными. Экстраполяция линейных зависимостей к $V \rightarrow 0$ позволяет получить истинные, методически неискаженные значения температур переходов.

На рис. 2 представлены подобные зависимости для исследованных образцов трикозана $(a)$, тетракозана $(b)$ и пентакозана $(c)$ в циклах нагревание-охлаждение. Полученные по этим зависимостям экстраполяционные (истинные) значения температур и гистерезисов фазовых переходов представлены в табл. 1. Из этих данных можно заключить, что в действительности для вторых максимумов температурный гистерезис в циклах нагревание-охлаждение отсутствует, а наблюдаемая на экспериментальных кривых ДСК разница в их положении на температурной шкале обусловлена методическими причинами. В то же время устранение методической погрешности с помощью экстраполяционных зависимо-
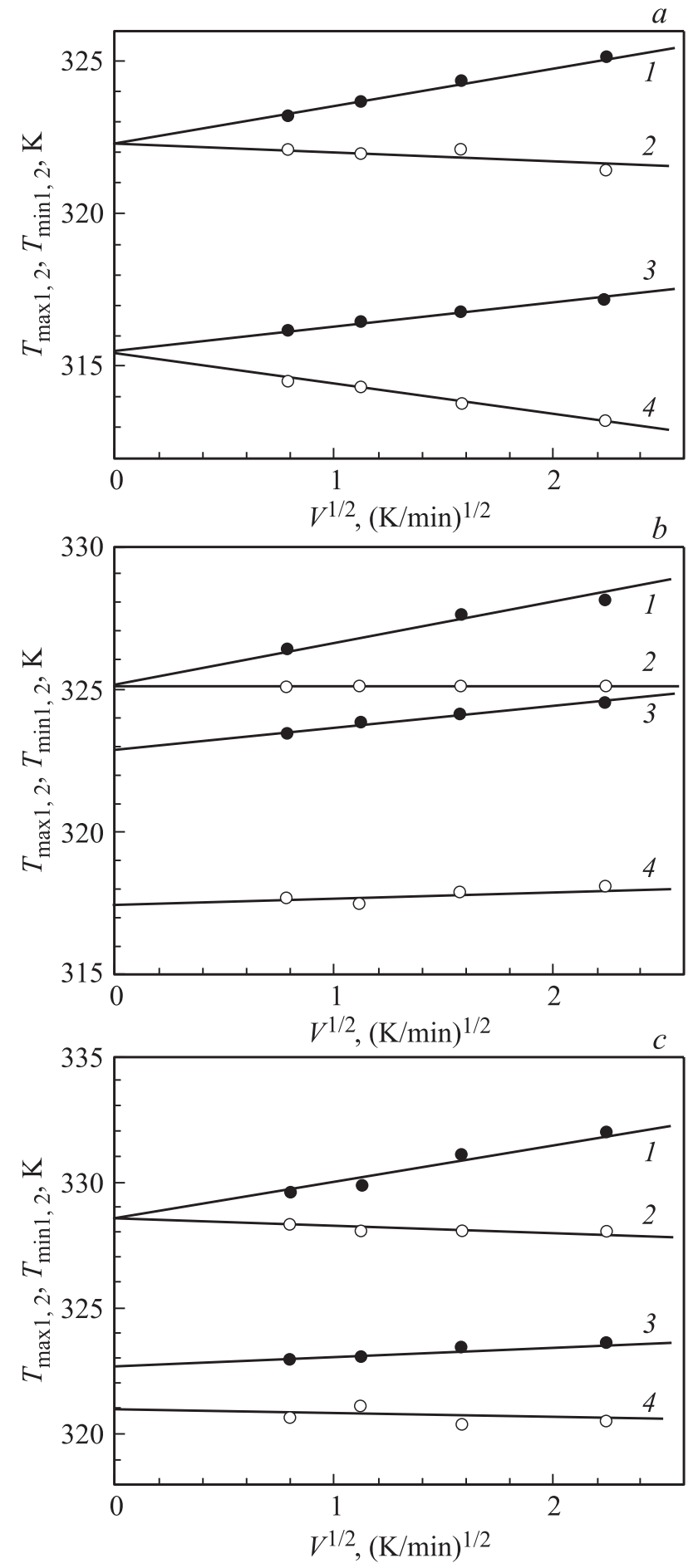

Рис. 2. Зависимости температур ФП от скорости сканирования для трикозана $(a)$, тетракозана $(b)$ и пентакозана $(c)$. Темные точки - нагревание, светлые - охлаждение. $1-T_{\max 2}$, $2-T_{\min 2}, 3-T_{\max 1}, 4-T_{\min 1}$.

стей позволило выявить для низкотемпературного перехода в этих образцах наличие истинного гистерезиса.

Энтальпия $\Delta H$ и энтропия $\Delta S$ переходов в методе ДСК не зависят от скорости сканирования по температуре и определяются соотношениями $\Delta H_{\exp }=\int C_{p}(T) d T$ и 
Таблица 1. Температурные параметры фазовых переходов в трикозане, тетракозане и пентакозане (по результатам экстраполяции к нулевой скорости сканирования)

\begin{tabular}{c|c|c|c|c|c|c|c}
\hline \multirow{2}{*}{ Образец } & \multirow{2}{*}{$\begin{array}{c}\text { Вид } \\
\text { сканирования }\end{array}$} & \multicolumn{3}{|c|}{ Пик 1 в дублете } & \multicolumn{3}{|c}{ Пик 2 в дублете } \\
\cline { 3 - 7 } & $T_{\max }, \mathrm{K}$ & $T_{\min }, \mathrm{K}$ & $\Delta T, \mathrm{~K}$ & $T_{\max }, \mathrm{K}$ & $T_{\min }, \mathrm{K}$ & $\Delta T, \mathrm{~K}$ \\
\hline \multirow{2}{*}{ Трикозан } & $\begin{array}{l}\text { Нагревание } \\
\text { Охлаждение }\end{array}$ & 315.4 & 314.9 & 0.5 & 322.3 & 322.3 \\
Петракозан & $\begin{array}{l}\text { Нагревание } \\
\text { Охлаждение } \\
\text { Пентакозан }\end{array}$ & 322.9 & 317.4 & 5.5 & 325.5 & 325.5 & 0 \\
$\begin{array}{l}\text { Нагревание } \\
\text { Охлаждение }\end{array}$ & 322.4 & 321.1 & 1.3 & 328.5 & 328.5 & 0
\end{tabular}

Таблица 2. Термодинамические параметры фазовых переходов в трикозане, тетракозане и пентакозане

\begin{tabular}{l|c|c|c|c|c|c}
\hline \multirow{2}{*}{ Образец } & $\begin{array}{c}\Delta H_{1}, \\
\mathrm{~J} / \mathrm{g}\end{array}$ & $\begin{array}{c}\Delta S_{1}, \\
\mathrm{~J} /(\mathrm{g} \cdot \mathrm{K})\end{array}$ & $\begin{array}{c}\Delta H_{2}, \\
\mathrm{~J} / \mathrm{g}\end{array}$ & $\begin{array}{c}\Delta S_{2}, \\
\mathrm{~J} /(\mathrm{g} \cdot \mathrm{K})\end{array}$ & $\begin{array}{c}\Delta H_{\text {summ }}, \\
\mathrm{J} / \mathrm{g}\end{array}$ & $\begin{array}{c}\Delta S_{\text {summ }}, \\
\mathrm{J} /(\mathrm{g} \cdot \mathrm{K})\end{array}$ \\
\hline Трикозан & 61 & 0.193 & 172 & 0.534 & 233 & 0.727 \\
Тетракозан & 89 & 0.276 & 153 & 0.470 & 242 & 0.746 \\
Пентакозан & 79 & 0.245 & 155 & 0.472 & 234 & 0.717
\end{tabular}

$\Delta S_{\exp }=\int C_{p}(T) d(\ln T)$. Суммарные значения энтальпии $\Delta H_{\text {summ }}$ и энтропии $\Delta S_{\text {summ }}$ и их составляющие для переходов первого рода $\left(\Delta H_{1}, \Delta S_{1}\right)$ и второго рода $\left(\Delta H_{2}\right.$, $\left.\Delta S_{2}\right)$ представлены в табл. 2.

Как известно [11], наличие или отсутствие температурного гистерезиса какого-либо физического свойства, в том числе для пиков на температурной зависимости теплоемкости, является одним из важных признаков, по которому ФП относят соответственно к переходам первого или второго рода. Поэтому отсутствие гистерезиса для вторых максимумов прямо указывает на принадлежность высокотемпературных пиков в дублетах к переходам второго рода. Если не использовать экстраполяционные зависимости $T_{\max , \min }=f\left(V^{1 / 2}\right)$, применяемые в настоящей работе, а пользоваться лишь данными, полученными при одной скорости сканирования по температуре (рис. 1), то высокотемпературные пики можно ошибочно отнести также к переходам первого рода, что, к сожалению, и делается в ряде публикаций. С другой стороны, наличие истинного гистерезиса для низкотемпературного пика позволяет на основании данных ДСК отнести этот переход к твердофазному (структурному) переходу первого рода, связанному с изменением типа симметрии кристалла. Ранее [2,14-16] методом рентгеновской дифракции было показано, что в температурном интервале, соответствующем пику теплоемкости с $T_{\max 1}$, действительно происходит изменение симметрии молекулярных кристаллов: орторомбическая элементарная субъячейка превращается в гексагональную в кристаллических сердечниках ламелей.
Если переходы первого и второго рода разнесены на температурной шкале, то в этом случае пик твердофазного перехода можно анализировать независимо от перехода второго рода. Как видно из рис. 1, эндо- и экзопики $C_{p}$ для исследуемых образцов, соответствующие ФП первого и второго рода, в цикле нагревание-охлаждение (переход из кристаллического состояния в расплав и обратный переход из расплава в кристалл) четко разнесены на температурной шкале. Это обстоятельство позволяет выделить эндотермические пики, отвечающие структурным ФП первого рода (рис. 3). Из рисунка видно, что форма пиков оказалась несимметричной, что может быть обусловлено наличием по крайней мере двух составляющих. Эти особенности формы пиков обсуждаются далее.

Анализ пиков, полученных указанным выше способом, проводился в рамках термодинамической теории самосогласованного поля $[9,10,17]$ применительно к $\Lambda$-образным размытым ФП первого рода. Тот факт, что переход становится размытым, означает, что из-

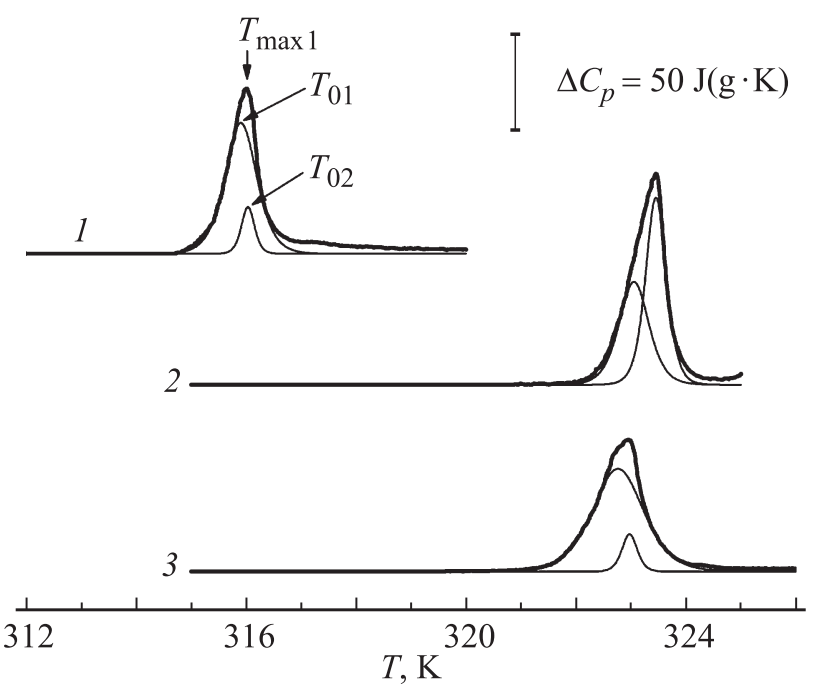

Рис. 3. Эндотермические пики, отвечающие твердофазным переходам в три- (1), тетра- (2) и пентакозане (3). Жирные линии - экспериментальные данные, тонкие линии - результат расчета по зависимости (1). 
Таблица 3. Термодинамические параметры фазового перехода первого рода в трикозане, тетракозане и пентакозане

\begin{tabular}{c|c|c|c|r|r|r|r}
\hline Образец & $\begin{array}{c}\text { Пик } \\
\text { в дублете }\end{array}$ & $\begin{array}{c}\text { Доля в энергии } \\
\text { перехода, \% }\end{array}$ & $T_{0}, \mathrm{~K}$ & $B$ & $\Delta C_{\max }, \mathrm{J} /(\mathrm{g} \cdot \mathrm{K})$ & $q_{0}, \mathrm{~J} / \mathrm{g}$ & $\omega, \mathrm{nm}^{3}$ \\
\hline Трикозан & 1 & 87 & 315.9 & 1650 & 70 & 54 & 128 \\
Тетракозан & 2 & 13 & 316.03 & 4000 & 25 & 5 & 2700 \\
& 1 & 44 & 323.05 & 1800 & 55 & 39 & 250 \\
Пентакозан & 2 & 56 & 323.45 & 2600 & 100 & 50 & 290 \\
& 2 & 91 & 322.76 & 1250 & 55 & 68 & 100 \\
& 2 & 9 & 322.97 & 3500 & 20 & 7 & 2800
\end{tabular}

менение фазового состояния происходит не внезапно, а постепенно и осуществляется в пределах определенного температурного интервала. Теория позволяет анализировать изменение в фазовом состоянии системы взаимодействующих частиц различных фаз, в частности образование и последующий рост устойчивых зародышей новой фазы в матрице старой фазы. Механизм нуклеации - гетерогенный, причем зародыши новой фазы возникают на различного типа дефектах в кристаллах, что приводит к появлению межфазной границы, которая является характерным признаком перехода первого рода. Основная идея теории состоит в том, что в объеме старой фазы происходит локализация многочисленных флуктуаций в ограниченном объеме в виде стабильных зародышей новой фазы - так называемых элементарных объемов перехода $\omega$. В дальнейшем по мере развития перехода движение межфазной границы осуществляется путем последовательных добавлений зародышей с объемом $\omega$ на возникшую межфазную границу. Образование зародышей-доменов требует изменения температуры на малую величину, которая определяется энергией, необходимой для возникновения таких областей. Это и приводит к размытию перехода по температуре. Например, для сегнетоэлектрических материалов было показано, что элементарные объемы превращения сопоставимы с объемом так называемой области Кенцига $\left(\sim 10^{-18}-10^{-17} \mathrm{~cm}^{3}\right)$ и по своему масштабу находятся на мезоскопическом уровне $[18,19]$.

Размеры устойчивых зародышей $\omega$ можно определить исходя из формы пиков $C_{p}$, соответствующих переходам первого рода. В работе [20] получено соотношение для температурной зависимости теплоемкости при размытом фазовом переходе в виде

$\Delta C_{p}(T)=4 \Delta C_{m} \exp \left[B\left(T-T_{0}\right) / T_{0}\right]\left[1+\exp \left[B\left(T-T_{0}\right) / T_{0}\right]^{-2}\right.$,

где $T_{0}$ - температура ФП первого рода, $\Delta C_{m}-$ максимальное значение теплоемкости при $T=T_{0}, B-$ атермический параметр.

В несимметричных пиках производилось разделение на два пика симметричной $\Lambda$-образной формы при условии равенства энтальпии экспериментально полученного пика сумме энтальпий двух симметричных пиков. Путем варьирования параметров $T_{0}, \Delta C_{\max }$ и $B$ для каждого из симметричных пиков можно было получить совпадение либо с правым (высокотемпературным), либо с левым (низкотемпературным) плечом пика. Наилучшее совпадение расчетных и экспериментальных зависимостей наблюдалось при значениях параметров, указанных в табл. 3 (пики 1 и 2). На рис. 3 показаны результаты расчета $\Lambda$-образных пиков по соотношению (1) и экспериментальные зависимости теплоемкости $\Delta C_{p}(T)$.

Параметр $B$ в соотношении (1) содержит наиболее интересную информацию о физической природе ФП, поскольку он связан с величиной пика теплоемкости $\Delta C_{m}$

$$
\Delta C_{m}=q_{0} B / 4 T_{0},
$$

где $q_{0}$ - теплота превращения, и элементарным объемом превращения $\omega$

$$
B=\omega \rho q_{0} / k T_{0},
$$

где $k-$ постоянная Больцмана, $\rho$ - плотность.

Параметр $B$ оказывается структурно-чувствительным, поскольку определяет в материалах с размытыми ФП объемы зародышей новой фазы [10]. Из соотношения (2) можно найти значения удельной теплоты (энтальпии) превращения, из соотношения (3) - элементарный объем превращения $\omega$ (полагая в первом приближении, что плотность кристаллов парафинов $\left.\rho \sim 0.8 \mathrm{~g} \cdot \mathrm{cm}^{-3}[1]\right)$. Результаты расчета по этим соотношениям приведены в табл. 3.

Для широкого круга молекулярных кристаллов н-алканов и их производных ранее было установлено [3-5], что элементарные объемы областей зародышеобразования новой фазы, связанные с твердотельным переходом, зависят от четности числа С-C-связей. Так, для четных $n$ объемы $\omega$ превышают в 2 раза объемы $\omega$ для нечетных $n$ и составляют соответственно величины $\sim 200$ и $\sim 100 \mathrm{~nm}^{3}$. Из сравнения значений $\omega$, приведенных в табл. 3 (пик 1), видно, что это соотношение в целом (для 87\% массы кристалла трикозана и 91\% пентакозана) выполняется. Оценка числа молекул, участвующих в образовании зародыша новой фазы в трикозане и пентакозане, показывает, что в элементарных объемах превращения $\left(\omega \sim 100-130 \mathrm{~nm}^{3}\right)$ на первой стадии фазового перехода содержится $\sim 200-250$ молекул, т. е. область превращения захватывает весьма большой домен. 
В тетракозане фазовый переход происходит также в две стадии (пики 1 и 2 в табл. 3), заметно различающиеся по величине элементарного превращения и содержащие $\sim 500-600$ молекул. Еще большее число молекул $(\sim 5000)$ участвует в образовании зародышей новой фазы на второй стадии фазового перехода в трикозане и пентакозане (пики 2 в табл. 3 ).

При рассмотрении возможных локальных мест возникновения устойчивых зародышей новой фазы необходимо учитывать форму и местоположение доменов или зародышей новой фазы. Из соображений минимизации внутренней энергии естественно предположить, во-первых, что зародыш новой фазы может располагаться в пределах стопки из двух ламелей, как это было показано в [21], а во-вторых, что фазовая граница не может проходить через часть молекулы, т. е. через наиболее сильные химические связи в метиленовой части молекул (C-C и $\mathrm{C}-\mathrm{H})$, и, по-видимому, проходит через относительно слабые ван-дер-ваальсовы связи между метиленовыми группами соседних молекул и концевыми группами $\mathrm{CH}_{3}$. Таким образом, домен можно представить в виде параллелепипеда, состоящего из параллельно упакованных „стержней“, в основании которых содержится 200-600 метиленовых цепей. Дальнейший рост объема новой фазы, как отмечалось выше, будет легче происходить за счет присоединения новых объемов к боковой поверхности. Этот рост будет происходить до тех пор, пока на боковой поверхности не появятся или не накопятся препятствия, увеличивающие боковую поверхностную энергию. В этом случае метиленовым группам необходимо преодолеть помимо сил межмолекулярного взаимодействия (ММВ) дополнительный барьер, препятствующий присоединению новых объемов к боковой поверхности.

В случае молекулярных кристаллов парафинов, как отмечалось выше, силами связи между структурными единицами являются силы ММВ. Для боковых поверхностей элементарного объема это сила ван-дерваальсового взаимодействия групп $-\mathrm{CH}_{2}-$. Для торцевых поверхностей (группы $-\mathrm{CH}_{3}-$ ) это тоже силы ван-дер-ваальсового взаимодействия. Количественной мерой ММВ является энергия когезии $E_{c}$, определяемая как энергия, необходимая для разрушения межмолекулярных контактов и перевода твердого тела через жидкое состояние в газообразную фазу. Уменьшение же энергии межмолекулярного взаимодействия при фазовом переходе происходит вследствие частичного уменьшения энергии когезии $\Delta E_{c}$. Экспериментально определить это уменьшение для веществ, состоящих из многоатомных молекул, затруднительно. $\Delta E_{c}$ можно сопоставить с величиной потенциального барьера движения молекулы в локальных жидкоподобных актах движения в твердом теле. Величина этого барьера, определенного экспериментальным путем для застеклованных жидкостей и твердых олигомеров, резко различающихся по характеру $\mathrm{MMB}$, строению и форме молекул, вклю- чая вещества с развитой системой водородных связей, составляет величину $\sim 0.4 E_{c}[22]$.

Экспериментально $E_{c}$ определяется только для низкомолекулярных тел, способных существовать в газовой фазе. Для большей части олигомеров, полимеров и молекулярных кристаллов процессу испарения предшествует деструкция, поэтому энергия когезии для них определяется косвенным путем либо рассчитывается [22,23]. В настоящей работе используются значения энергии когезии, приведенные в монографии [22] для вкладов от $\mathrm{CH}_{2}$-групп: $E_{c 1}=3.6 \mathrm{~kJ} / \mathrm{mol}$.

Как видно из рис. 3, на первой стадии происходит фазовое превращение, инициированное зарождением доменов новой фазы размером $\omega \sim 100-130 \mathrm{~nm}^{3}$ с преодолением барьера ММВ, равного $0.4 E_{c 1}$. Фазовое превращение в большей части кристалла (это следует из соотношения величин теплот и превращения на первой и второй стадиях, табл. 3) приводит к исчерпанию бездефектных областей кристалла и повышению поверхностной энергии вследствие накопления или появления препятствий в оставшейся, т.е. не претерпевшей фазового превращения части кристалла. На второй стадии фазового перехода в трикозане и пентакозане с $\omega \cong 2700-2800 \mathrm{~nm}^{3}$ происходит, по-видимому, фазовое превращение в оставшейся части кристалла, связанное с преодолением появившихся и накопившихся препятствий.

Для оценки потенциального барьера, создаваемого этими препятствиями, обратимся к зависимости, связывающей один из геометрических параметров - толщину ламели $L-$ с величиной элементарного акта превращения $\omega$ :

$$
L=\omega^{1 / 3}\left(E_{c} / E_{c 1}\right)^{2 / 3},
$$

где $E_{c 1}$ и $E_{c}-$ энергии когезии метиленовой и концевой групп соответственно [21].

Из предположения о том, что дальнейший рост объема новой фазы при преодолении дополнительного барьера будет происходить за счет присоединения новых объемов к боковой поверхности, т. е. без изменения толщины стенки, следует неизменность величины $E_{c}$. Последнее позволяет получить из выражения (4) соотношение

$$
E_{c 2}=\omega_{2}^{1 / 2} \omega_{1}^{-1 / 2} E_{c 1}
$$

и определить искомую величину потенциального барьера, равную $\sim 0.4 E_{c 2}$. Для трикозана, тетракозана и пентакозана эти величины оказались равны $6.6 \mathrm{~kJ} / \mathrm{mol}(1.6 \mathrm{kcal} / \mathrm{mol}), 3.9 \mathrm{~kJ} / \mathrm{mol}(0.4 \mathrm{kcal} / \mathrm{mol})$ и $7.6 \mathrm{~kJ} / \mathrm{mol}(1.8 \mathrm{kcal} / \mathrm{mol})$ соответственно.

Сопоставление полученных значений с известными из литературы данными по парафинам [24] позволяет трактовать эти потенциальные барьеры как барьеры, препятствующие движению вдоль оси молекулы молекулярных дефектов типа кинков $2 g 1$ по механизму флип-флоп. Эти дефекты, движение которых связано со смещением на одну группу $-\mathrm{CH}_{2}-\mathrm{c}$ одновременной 
ротацией транс-зигзага на $180^{\circ}$, имеют два потенциальных барьера. Один из них $(\sim 1.7 \mathrm{kcal} / \mathrm{mol})$ препятствует смещению выступающей в межламелярную прослойку концевой группы $-\mathrm{CH}_{3}-$ и движению макромолекулы парафина внутрь ламелярного сердечника, другой $(\sim 0.6 \mathrm{kcal} / \mathrm{mol})$ - в противоположном направлении.

В работе [25] показано, что при нагревании н-алканов (особенно в области фазовых переходов) резко возрастает число нерегулярных конформеров типа кинков $2 g 1$ и концевых гош-конформеров типа $g-t_{m}$, где $t_{m}-$ транс-последовательность из $\mathrm{CH}_{2}$-групп при $m \geq 5$.

Появление этих нерегулярных конформеров приводит к нарушению первоначального строения кристаллической сердцевины ламелей н-алканов, что, естественно, происходит при фазовом переходе первого рода.

Мы полагаем, что эти конформационные дефекты в процессе развития фазового перехода концентрируются преимущественно на фазовых границах возникающих зародышей, тем самым создавая препятствия для дальнейшего распространения границы. Для преодоления возникающих потенциальных барьеров необходима дополнительная энергия, обеспечиваемая последующим повышением температуры.

\section{Список литературы}

[1] D.M. Small. The physical chemistry of lipids. Plenum Press, N. Y.-London (1986). 262 p.

[2] T. Yamamoto, K. Nozaki, T. Hara. J. Phys. Chem. 92, 631 (1990).

[3] В.М. Егоров, В.А. Марихин, Л.П. Мясникова, N. Nakamura. ФTT 51, 2006 (2009).

[4] В.М. Егоров, В.А. Марихин, Л.П. Мясникова. Высокомолекуляр. соединения 53, 1722 (2011).

[5] В.М. Егоров, В.А. Марихин, Л.П. Мясникова. ФТТ 55, 975 (2013).

[6] Y. Ogawa, N. Nakamura. Bull. Chem. Soc. Jpn. 72, 943 (1999).

[7] В.М. Егоров, В.А. Марихин, Л.П. Мясникова. Высокомолекуляр. соединения А 48, 2138 (2006).

[8] В.М. Егоров, В.А. Марихин. ФТТ 58, 2482 (2016).

[9] Б.Н. Ролов, В.Э. Юркевич. Физика размытых фазовых переходов. Изд-во Ростов. ун-та, Ростов (1983). 317 с.

[10] Г.А. Малыгин. УФН 171, 187 (2001).

[11] Л.Д. Ландау, Е.М. Лифшиц. Статистическая физика. Наука, M. (1976). $514 \mathrm{c}$.

[12] V.A. Bershtein, V.M. Egorov. Differential scanning calorimetry of polymers: physics, chemistry, analysis, technology. Ellis Horwood, N. Y. (1994). 253 p.

[13] K. Illers. Eur. Polym. J. 10, 911 (1974).

[14] S. Abrahamsson, G. Larsson, E. Sydov. Acta Cryst. 13, 770 (1960).

[15] R. Popovitz-Biro, J.L. Wang, J. Majewski, E. Shavit, L. Leiserowitz, M. Lahav. J. Am. Chem. Soc. 116, 1179 (1994).

[16] J-L. Wang, F. Leveiller, D. Jacqueman, K. Kjaer, J. Als-Nielsen, M. Lahav, L. Leiserowitz. J. Am. Chem. Soc. 116, 1192 (1994).

[17] М. Фишер. Природа критического состояния. Мир, М. (1968). $221 \mathrm{c}$.
[18] В. Кенциг. Сегнетоэлектрики и антисегнетоэлектрики. ИЛ, М. (1960). 347 с.

[19] В.Я. Фрицберг. Изв. АН СССР. Сер. физ. 47, 698 (1983).

[20] Г.А. Малыгин. ФТТ 43, 1911 (2001).

[21] В.М. Егоров, В.А. Марихин. ФТТ 58, 2482 (2016).

[22] D.W. Van Krevelen. Properties of polymers correlations with chemical structure. N.Y. (1972) $480 \mathrm{p}$.

[23] А.А. Аскадский, Л.К. Колмакова, А.А. Тагер, Г.Л. Слонимский, В.В. Коршак. Высокомолекуляр. соединения А $\mathbf{1 9}$, 1004 (1977).

[24] G. Strobl, B. Even, E.W. Fischer, W. Piesczek. J. Chem. Phys. 61, 5257 (1974).

[25] R.G. Snyder, M. Maroucelli, S.P. Qi, H.L. Strauss. Science 214, 188 (1981) 